\title{
Removal of Motion Artifacts from ECG signals by Combination of Recurrent Neural Networks and Deep Neural Networks
}

\author{
$1^{\text {st }}$ Muhammad Zubair \\ Department of Electronics and Communication Engineering \\ Prasad V. Potluri Siddhartha Institute of Technology \\ Vijayawada, Andhra Pradesh, India \\ muhammadzub17@gmail.com
}

\author{
$2^{\text {nd }}$ Gunturi N V S Chandra Mouli \\ Department of Electrical and Electronics Engineering \\ Prasad V. Potluri Siddhartha Institute of Technology \\ Vijayawada, Andhra Pradesh, India \\ gunturicm@gmail.com
}

\author{
$3^{\text {rd }}$ Rafi Ahamed Shaik \\ Department of Electronics and Electrical Engineering \\ Indian Institute of Technology Guwahati \\ Guwahati, Assam, India \\ rafiahamed@iitg.ac.in
}

\begin{abstract}
Electrocardiogram (ECG) is the graphical portrayal of heart usefulness. The ECG signals holds its significance in the discovery of heart irregularities. These ECG signals are frequently tainted by antiques from various sources. It is basic to diminish these curios and improve the exactness just as dependability to show signs of improvement results identified with heart usefulness. The most commonly disturbed artifact in ECG signals is Motion Artifacts (MA). In this paper, we have proposed a new concept on how machine learning algorithms can be used for de-noising the ECG signals. Towards the goal, a unique combination of Recurrent Neural Network (RNN) and Deep Neural Network (DNN) is used to efficiently remove MA. The proposed algorithm is validated using ECG records obtained from the MIT-BIH Arrhythmia Database. To eliminate MA using the proposed method, we have used Adam optimization algorithm to train and fit the contaminated ECG data in RNN and DNN models. Performance evaluation results in terms of SNR and RRMSE show that the proposed algorithm outperforms other existing MA removal methods without significantly distorting the morphologies of ECG signals.

Index Terms-Electrocardiogram (ECG), Motion Artifacts (MA), Adam optimization algorithm, Recurrent Neural Networks (RNN), Deep Neural Networks (DNN)
\end{abstract}

\section{INTRODUCTION}

The electrocardiogram is a non-obtrusive demonstrative device to record the electrical action of the heart. This is practised by estimating the possible distinction between a few terminals that are set on the skin at predefined purposes of the human body. One pattern of the Electrocardiographic (ECG) speaks to the depolarization/re-polarization of the chamber and the ventricle which happens for each heartbeat. ECG signals are composed of a train of segments $\mathrm{P}, \mathrm{QRS}$, and T separated by iso-electric regions as shown in Fig. 1. The ECG signals are frequently sullied by motion artifacts. The ECG signal is a broadly utilized analytic instrument for investigation and

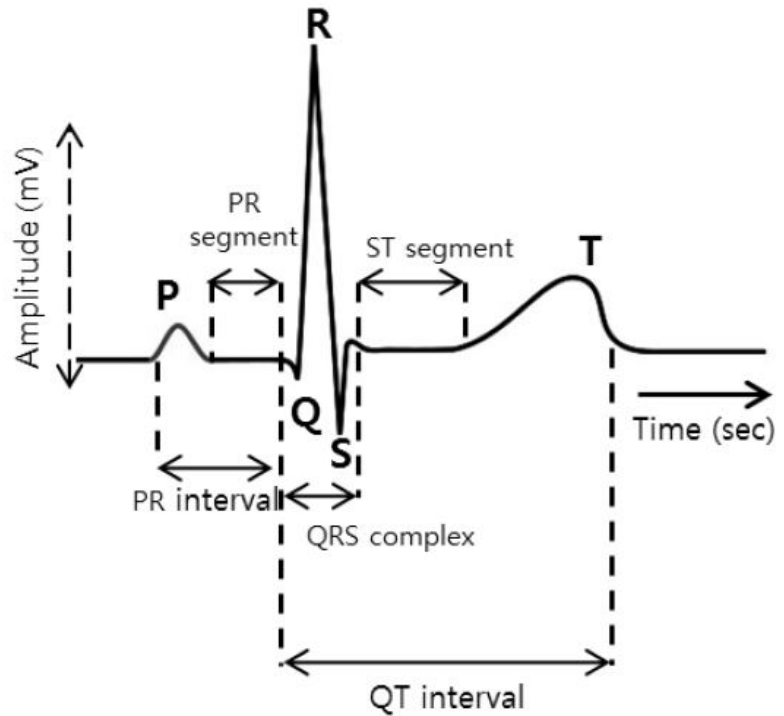

Fig. 1. Single cardiac cycle of ECG signal

conclusion of heart anomalies identified with various kinds of arrhythmias. In most clinical assessment cases, the heart finding must be made all the more precisely. In this manner, the recognition and expulsion of MA from the ECG signal represents a genuine test and is urgent for the solid understanding of ECG-based quantitative measures. Various strategies have been produced for detection and expulsion of antiques in the ECG and EEG signals [19]- [21], these strategies depend on the signal processing methods including, empirical mode decomposition (EMD) [1]- [2], singular value decomposition (SVD) filter [3], morphological operators [4], independent 
component analysis (ICA) [5], ensemble empirical mode decomposition (EEMD) [6]. In [7], $\mathrm{Li}$ and Lin presented an ideal de-noising calculation for ECG signals which utilizes the fixed wavelet change (SWT). In spite of the fact that this strategy is effective in evacuating invariant commotion this calculation isn't vigorous against motion incited relics and impulsive moises.

Although in the works mentioned above various algorithms have been widely studied, the performance of MA from ECG signals can be further improved by considering deep learning algorithms. As of late, deep learning has been applied to numerous fields in biomedical engineering. In [8], convolutional neural systems $(\mathrm{CNN})$ are utilized to discover ectopic beats, which accomplish a better presentation over the vast majority of different calculations. In addition, recurrent neural networks (RNN) are used in [9] to classify ECG beats and in [10] lyapunouv exponents are fed into RNN to classify beats. Earlier exploration has been led to the utilization of ML in denoising signals. In [11] by Lipsa Routray, Pradyut Biswal, and Satya Ranjan Pattanaik, they utilized Functional Link Adaptive Neural Network (FLANN) and Radial Basis Functional Network (RBFN) to expel ECG defilement from EEG signal. In [12] Nebras.A.Mohamed et.al utilized Artificial Neural Network (ANN) to analyze cardiovascular issues utilizing ECG signals and for the reason for denoising, they utilized Bandpass and Bandstop channels. In [13] Manish N. Tibdewal and Kanchan B. Thakare utilized ANN to group visual ancient rarities (Eyeblink (EB) and Eye development artifacts (EMA)) and non - artifactual EEG. In [14], a paper was distributed by Monalisa Singha Roy et.al on recognizing motion antiquities (MA) and redressing beat morphology from Photoplethysmography (PPG) utilizing Artificial Neural Network (ANN). In [15] authors proposed detection of first stage of atrioventricular block (I-AVB) from electrocardiogram (ECG) signals.

Similarly, to remove the MA from the ECG signals in a more efficient manner we have proposed a hybrid deep learning model which is the combination of recurrent neural network (RNN) and deep neural network (DNN). In the proposed method we have trained the RNN-based model using the noisy ECG data using Adam optimization algorithm in order to obtain the artifact free ECG signal. Secondly, filtered signal from the RNN model is used to train the DNN model to obtain completely clean ECG signal with a very high accuracy of noise reduction when compared to state-of-theart techniques.

The further segments of this paper are definite and composed as follows: Section 2 presents the detailed explanation of the algorithms applied in our work. Section 3 frameworks the architecture of proposed RNN-DNN models used to reject motion artifacts in ECG signals. Section 4 shows the test results and perceptions that have been gotten. At long last, the last area gives the ends and clarifies the potential future work that could be performed upon the proposition.

\section{Algorithm}

\section{A. Adam Optimization Algorithm}

The stochastic gradient (or) slope based optimization algorithm increased down to earth significance in numerous fields of science and engineering. Numerous issues in these fields can be given a role as the optimization of some scalar parametrized target work requiring augmentation or minimization concerning its boundaries. On the off chance that the capacity is differentiable regarding its boundaries, gradient decent is a moderately productive optimization technique, since the calculation of first-order fractional subordinates as for all the boundaries is of a similar computational multifaceted nature as simply assessing the capacity [16].

For efficient stochastic optimization using only first-order gradients with little memory requirements, Kingma etal. proposed Adam algorithm as shown in Algorithm 1 [16]. This algorithm computes individual adaptive learning rates for different parameters from estimates of first and second moments of the gradients.

Algorithm 1 Adam algorithm for stochastic optimization. In the calculation utilized beneath $g_{t}^{2}$ demonstrates the component savvy square $g_{t} \odot g_{t}$. Great default settings for the tried ML issues are $\alpha=0.001, \beta_{1}=0.9, \beta_{2}=0.999$ and $\epsilon=10^{-8}$. All procedure on vectors are component shrewd. With $\beta_{1}^{t}$ and $\beta_{2}^{t}$ we indicate $\beta_{1}$ and $\beta_{2}$ to the power $t$.

Require: $\alpha$ : Step size

Require: $\beta_{1}, \beta_{2} \in(0,1)$ : Exponential decay rates for the moment estimates

Require: $f(\theta)$ :Stochastic objective function parameters $\theta$

Require: $\theta_{o}$ : Initial parameter vector

$m_{o} \leftarrow 0$ (Initialize 1st moment vector)

$v_{o} \leftarrow 0$ (Initialize 2nd moment vector)

$t \leftarrow 0$ (Initialize time step)

while $\theta_{t}$ not converged do

$t \leftarrow t+1$

$g_{t} \leftarrow \nabla_{\theta} f_{t}\left(\theta_{t-1}\right)$ (Get gradients w.r.t. stochastic objective at time step $t)$

$m_{t} \leftarrow \beta_{1} \cdot m_{t-1}+\left(1-\beta_{1}\right) \cdot g_{t}$ (Update biased first moment estimate)

$v_{t} \leftarrow \beta_{2} \cdot v_{t-1}+\left(1-\beta_{2}\right) \cdot g_{t}^{2}$ (Update biased second raw moment estimate)

$\hat{m}_{t} \leftarrow m_{t} /\left(1-\beta_{1}^{t}\right)$ (Compute bias-corrected first moment estimate)

$\widehat{v}_{t} \leftarrow v_{t} /\left(1-\beta_{2}^{t}\right)$ (Compute bias-corrected second raw moment estimate)

$\theta_{t} \leftarrow \theta_{t-1}-\alpha \cdot \hat{m}_{t} /\left(\sqrt{\hat{v}_{t}}+\epsilon\right)$ (Update parameters)

end while

return $\theta_{t}$ (Resulting parameters)

\section{B. Deep Recurrent Neural Networks (DRNN)}

In the Recurrent Neural Network (RNN), another memory module is acquainted with contemplate history data in the 
characterizing procedure. To have long access to the past setting,Long Short Term Memory (LSTM) organize was proposed and has been the most comprehensively used intermittent structure up until this point. The structure of the LSTM memory square shows up in Fig. 2. Considering the commitment at time $t$ and the yield of memory obstructs at time $t-1$, the input door and the output gate accept accountability for when to send the data into or to examine the data from the memory square. Then, the forget gate gives a path to the memory cells to reset themselves. The heaps of the three gates and the memory cell are discovered during the readiness strategy. [17].

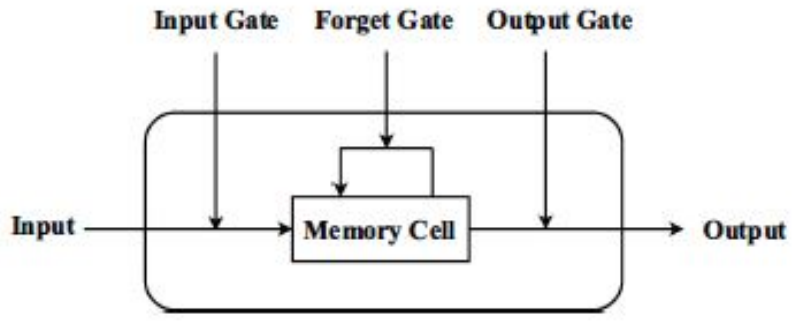

Fig. 2. LSTM internal structure

In the proposed technique Deep RNN (DRNN) is applied for noise separation as a regression problem which de-mixes the noisy signal $x_{t}$ into the de-noised signal $y_{t}$ at each time $t$. The relation between input signal $x_{t}$ and the $k^{t h}$ output node $y_{t k}$ can be formulated as

$$
\begin{aligned}
y_{t k} & =y_{k}\left(\mathbf{x}_{t}, \mathbf{w}\right) \\
& =f\left(\left(\mathbf{w}^{(2)}\right)^{\top} f\left(\left(\mathbf{w}^{(1)}\right)^{\top} \mathbf{x}_{t}+\left(\mathbf{w}^{(11)}\right)^{\top} \mathbf{z}_{t-1}\right)\right) \\
& =f\left(\left(\mathbf{w}^{(2)}\right)^{\top} \mathbf{z}_{t}\right) \\
& =f\left(a_{t k}\right)
\end{aligned}
$$

where the boundary set $\mathrm{w}$ comprises of input-to-hidden weight $\mathbf{w}^{(1)}$, hidden-to-hidden weights $\mathbf{w}^{(l, l-1)}$ and hidden-tooutput weights $\mathbf{w}^{(L)}$. The hidden-to-hidden weights $\mathbf{w}^{(l, l-1)}$ are otherwise called the recurrent weights. Here $L$ is spoken to as absolute number of layers in the system and $l$ is the quantity of current layer and is given by $l=(1,2,3, \ldots L)$. The visual depiction of the layers utilized in the DRNN model is appeared in Fig. 3.

\section{Deep Neural Networks (DNN)}

Deep learning dependent on the artificial neural network is run under a deep engineering or chain of command, comprising of numerous concealed layers, which catches the elevated level deliberation behind information and describes the complex non-straight connection among data sources and focuses as appeared in Fig. 4. An improved form of Deep Neural Network (DNN) can be spoken to as a various leveled

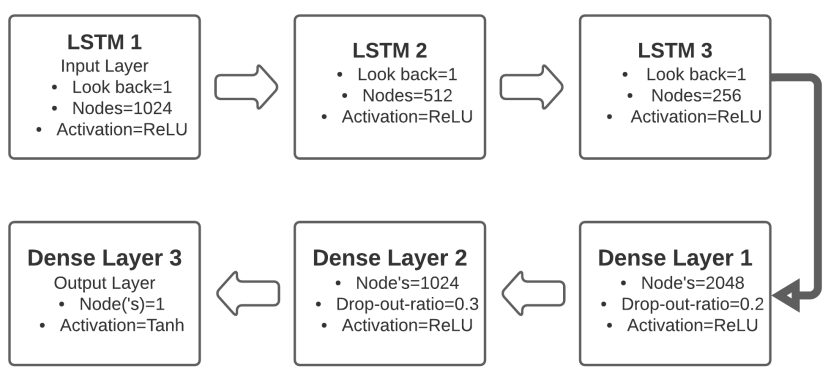

Fig. 3. Layer description of RNN model

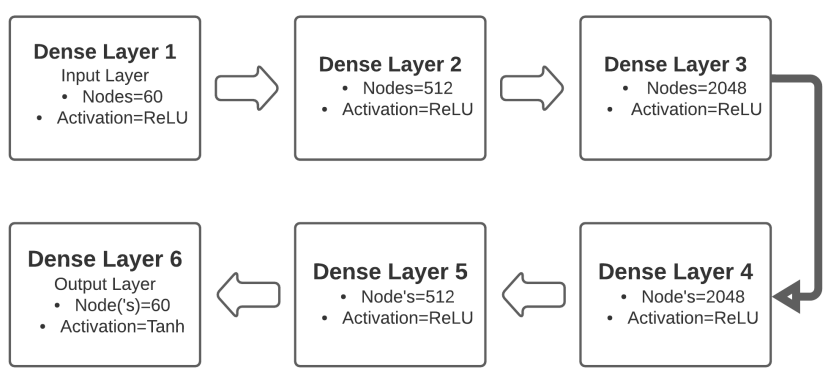

Fig. 4. Layers description of DNN model

association of neurons interconnected with one another as appeared in Fig. 5. These neurons pass a sign to different neurons dependent on the got info and structure an unpredictable system that learns with some criticism instrument [17]. In this paper, the DNN model appeared in Fig. 4 is utilized to expel MA from the polluted ECG signal.

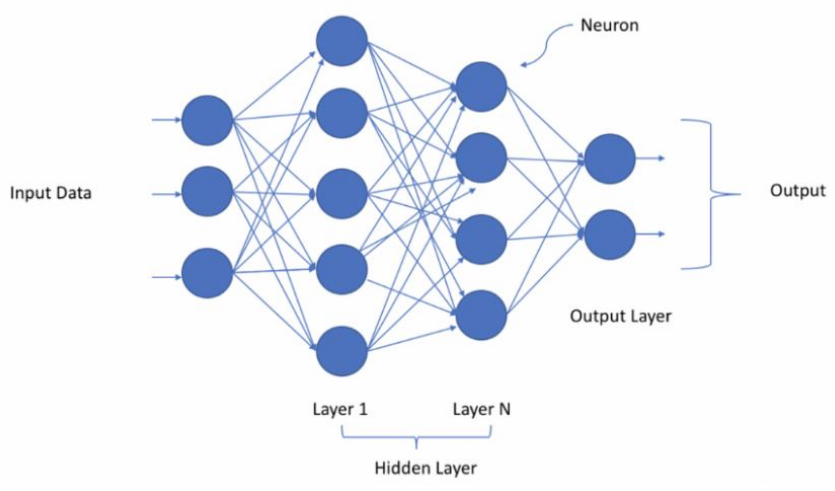

Fig. 5. Standard DNN model [23]

\section{Proposed Method:Combination of RNN-DNN MODELS TO ELIMINATE MOTION ARTIFACTS IN ECG SIGNALS}

In the proposed method we exploited the concept of machine learning algorithms for removing motion artifacts from the ECG signals. To execute this task an unique combination of RNN and DNN models are combined together to achieve 
TABLE I

NETWORK DESCRIPTION OF BOTH RNN AND DNN MODELS

\begin{tabular}{|l|l|l|l|l|}
\hline \multirow{2}{*}{$\begin{array}{l}\text { Layer } \\
\text { Number }\end{array}$} & \multicolumn{2}{|c|}{ Intermediate filter } & \multicolumn{2}{c|}{ Follow-up filter } \\
\cline { 2 - 5 } & $\begin{array}{l}\text { Node } \\
\text { Count }\end{array}$ & $\begin{array}{l}\text { Activation } \\
\text { Function }\end{array}$ & $\begin{array}{l}\text { Node } \\
\text { Count }\end{array}$ & $\begin{array}{l}\text { Activation } \\
\text { Function }\end{array}$ \\
\hline $\begin{array}{l}1 \\
\text { input } \\
\text { layer }\end{array}$ & $\begin{array}{l}1024 \\
\text { (LSTM) }\end{array}$ & ReLu & $\begin{array}{l}60 \\
\text { Dense }\end{array}$ & ReLu \\
\hline $\begin{array}{l}2 \\
\text { hidden } \\
\text { layer }\end{array}$ & $\begin{array}{l}512 \\
\text { (LSTM) }\end{array}$ & ReLu & $\begin{array}{l}512 \\
\text { Dense }\end{array}$ & ReLu \\
\hline $\begin{array}{l}3 \\
\text { hidden } \\
\text { layer }\end{array}$ & $\begin{array}{l}256 \\
\text { (LSTM) }\end{array}$ & ReLu & $\begin{array}{l}2048 \\
\text { Dense }\end{array}$ & ReLu \\
\hline $\begin{array}{l}4 \\
\text { hidden } \\
\text { layer }\end{array}$ & $\begin{array}{l}2048 \\
\text { (Dense) }\end{array}$ & ReLu & $\begin{array}{l}2048 \\
\text { Dense }\end{array}$ & ReLu \\
\hline $\begin{array}{l}5 \\
\text { hidden } \\
\text { layer }\end{array}$ & $\begin{array}{l}1024 \\
\text { (Dense) }\end{array}$ & ReLu & $\begin{array}{l}512 \\
\text { Dense }\end{array}$ & ReLu \\
\hline $\begin{array}{l}\text { output } \\
\text { layer }\end{array}$ & $\begin{array}{l}1 \\
\text { (Dense) }\end{array}$ & Tanh & $\begin{array}{l}60 \\
\text { Dense }\end{array}$ & Tanh \\
\hline
\end{tabular}

accurate results. Both RNN and DNN models in the proposed technique are trained using Adam optimization algorithm.

The proposed method contains the following four main steps:

- Train the RNN-based model with noisy ECG data.

- Use the trained RNN-based model to extract the artifacts present in the input ECG signal. The purpose of using an RNN-based model is to have an intermediate filter that learns how the data should progress over the time domain.

- Train DNN-based model with the filtered output taken from the RNN model.

- Use the trained DNN-based model to acquire completely artifact free ECG signal with high accuracy. This second stage of artifact removal is known as a follow-up network.

The complete architecture or hierarchy of the proposed method is shown in Fig. 6. The description of layers used in RNN and DNN is shown in Tabel I.

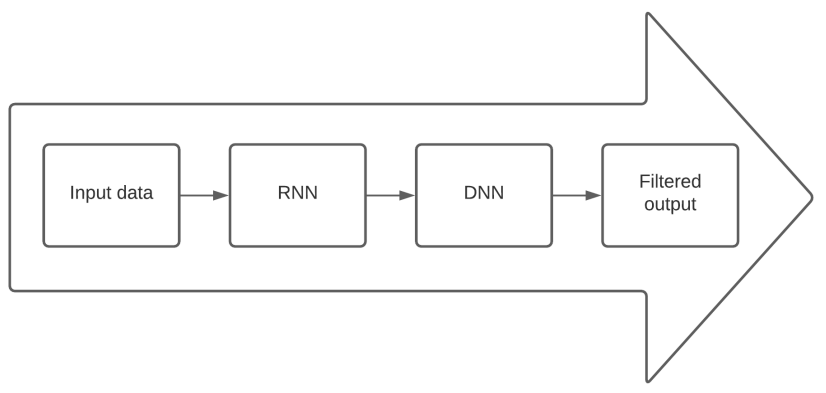

Fig. 6. Proposed RNN-DNN model

\section{EXPERIMENTAL RESULTS}

\section{A. Data Description}

The MIT-BIH Arrhythmia Database [22] contains 48 thirty minutes selections of two-channel wandering ECG recordings, got from 47 subjects by the BIH Arrhythmia Laboratory. Twenty-three chronicles were picked indiscriminately from a lot of 4000, 24-hour mobile ECG accounts gathered from a blended populace of inpatients (about 60\%) and outpatients (about 40\%) at Boston's Beth Israel Hospital; the staying 25 chronicles were chosen from a similar set to incorporate more uncommon yet clinically huge arrhythmias that would not be very much spoken to in a little arbitrary example. To perform the experiment using the proposed technique data was taken for patients (100-109). To train the RNN model data of 8 subjects was used $(100,101,102,103,106,107,108,109)$ and to test the results 2 subjects (104-105) data was used.

\section{B. MA removal using $R N N$-based Model}

Firstly, the ECG signal acquired from the MIT-BIH Arrhythmia database was added with some random noise. Secondly, at the initial stage of artifact removal we have used a RNNbased model to train and fit the contaminated ECG data so as to obtain a noise free ECG signal. We considered an RNN model with six layer network out of which the first three layers are LSTM layers with a node count of 1024, 512 and 256 respectively and the last three layers are regular dense layers with a node count of 2048, 1024 and 1 respectively. Upon which the first 2 dense layers have drop-out percentage of $20 \%$ and 30\% respectively. The data acquired from the database was digitized at 360 samples per second with each set 10 seconds interval and it was a dual channel data that means each set consists of total $7200(360 \times 10 \times 2)$ samples. For training the RNN model a total of 8 such sets were taken and 2 sets were kept for validation. The training data for the RNN model is prepared by stacking the data of all 8 sets on a single axis (1D array), thus the resulting dataset was of 57,600 $(7200 \times 8)$ samples and has only one feature per sample which is the amplitude value.

The RNN's memory was set for only 1 time-step, which means that only the previous time-step is remembered for the next time step. The training was done using Adam optimization algorithm with Log-Cosh as a loss function as described in (2).

$$
L\left(y, y^{p}\right)=\sum_{i=1}^{n} \log \left(\cosh \left(y_{i}^{p}-y_{i}\right)\right)
$$

where, $y$ represents original ECG signal and $y^{p}$ represents network predicted output. Finally, the training was stopped when the model has reached a relatively low loss value.

\section{Final Stage of MA removal using DNN-based Model}

In the second and final stage of artifact removal, we have used a regular DNN-based model to train and fit the filtered output data extracted from the RNN model. This second stage artifact removal technique is known as follow-up network. For 

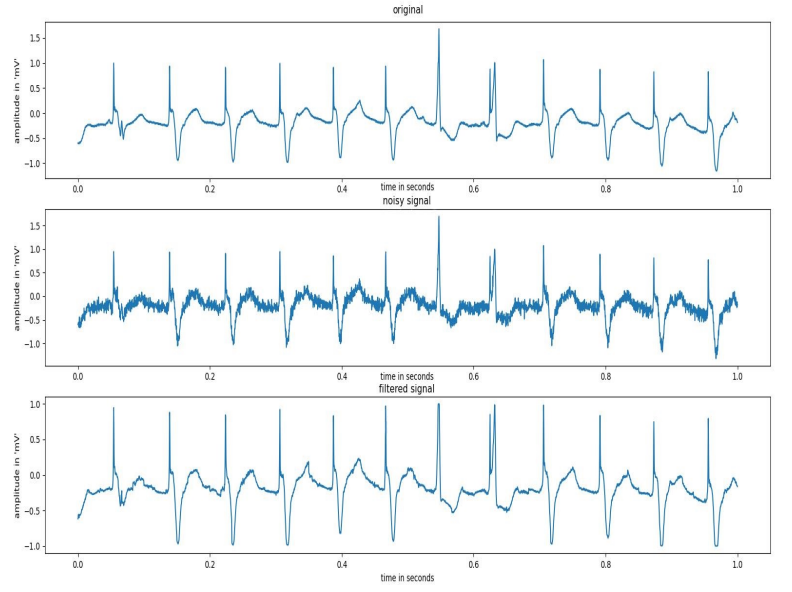

Fig. 7. Proposed RNN-DNN output

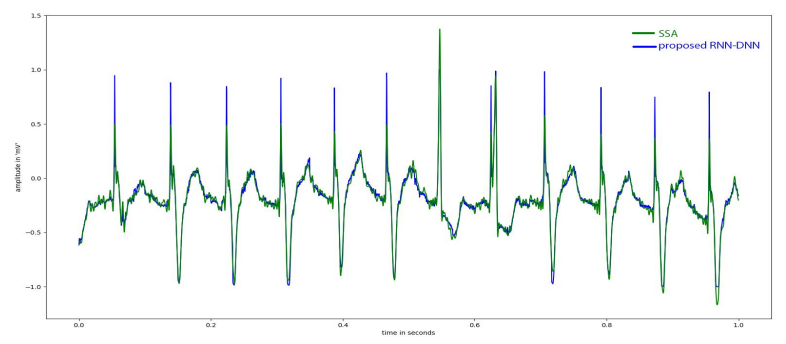

Fig. 8. Filtered ECG signal comparison between SSA and proposed RNNDNN

this model the weights of the saved RNN model are locked and are not allowed for further training, thus creates a rigidity in the first part of the whole artifact removal process. Since the weights of the RNN model are locked we have allowed the follow-up network to train and get better results than the first network. The follow-up network consists of 6 layers with each layer having a node count of 60, 512, 2048, 2048, 512 and 60 respectively. This enables the network to properly remap the output of the intermediate MA removal stage to the desired output. The neural network is fed with 60 features at a time and is enabled to give output of 60 filtered features. To perform this we re-stacked the output of the intermediate filter and the original samples to form a $[960 \times 60]$ matrix each. So, the DNN model is trained in such a way that the network takes 60 samples from the RNN's output data and tries to map them to the corresponding artifact free data samples. The output of the proposed method is shown in Fig. 7. Similar to the previous stage this model is also trained using Adam optimization algorithm and we have used Log-Cosh as loss function as mentioned in (2).

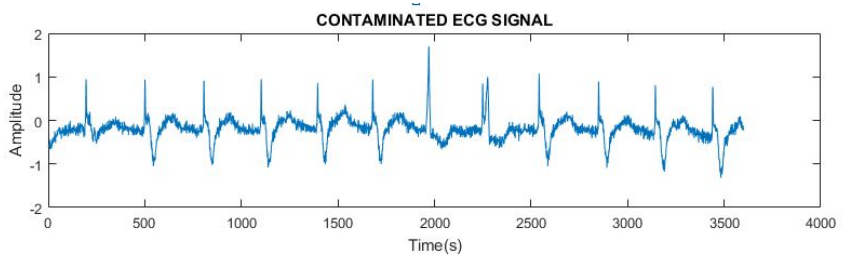

Fig. 9. Contaminated ECG signal

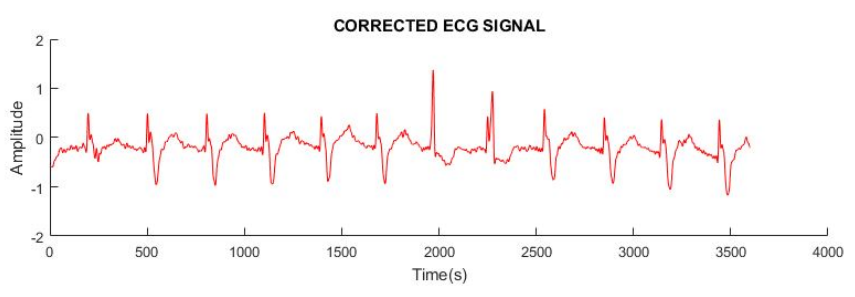

Fig. 10. Corrected ECG signal using spectrum analysis

\section{MA removal using SSA technique}

To compare the performance of the proposed technique we have implemented existing SSA technique for removing the motion artifacts from ECG signal. Firstly, the SSA method was used to decompose the ECG signal contaminated by the motion artifacts into a trajectory matrix by applying singular value decomposition (SVD) and the peak frequencies of each eigen vectors were calculated. Secondly, according to the difference between the frequency range of the ECG and the motion artifacts, high power frequencies related to the motion artifacts are compressed. Finally, the remaining data was reconstructed using diagonal averaging to obtain the clean ECG signal. The detailed algorithm of SSA technique is explained in [18]. The outputs obtained after implementing SSA technique is shown in Fig. 9 and Fig. 10. To visualize the performance of both the techniques artifact free ECG signals are plotted as shown in Fig. 8.

\section{E. Calculating SNR and RRMSE Values}

To demonstrate the performance of the proposed RNN-DNN technique, we have consider the mixing model as shown in (3)

$$
X(n)=S(n)+\rho A(n)
$$

where $S(n)$ is the source ECG signal, $A(n)$ is the noisy signal and $\rho$ is the propogation constant that will choose the SNR of the deliberate signal. The SNR of the deliberate sign $X(n)$ is given by

$$
S N R=\frac{R M S(S)}{R M S(\rho A)}
$$

where $R M S(S)$ is the root mean square of the ideal source signal. To look at the exhibition of the proposed procedure with the current otherworldly spectral analysis technique, for 
example, SSA, we have considered the relative root-meansquare error (RRMSE) as a parametric measure. The RRMSE of the ideal source signal $S(n)$ is given by

$$
R R M S E=\frac{R M S(S-\hat{S})}{R M S(S)} \times 100 \%
$$

Where $\mathbf{S}$ is the original data matrix and $\hat{\mathbf{S}}$ is the output data matrix of RNN-DNN model.

Finally, after calculating SNR and RRMSE values graph is plotted as shown in Fig. 11 to compare the performance of the proposed technique with the existing technique.

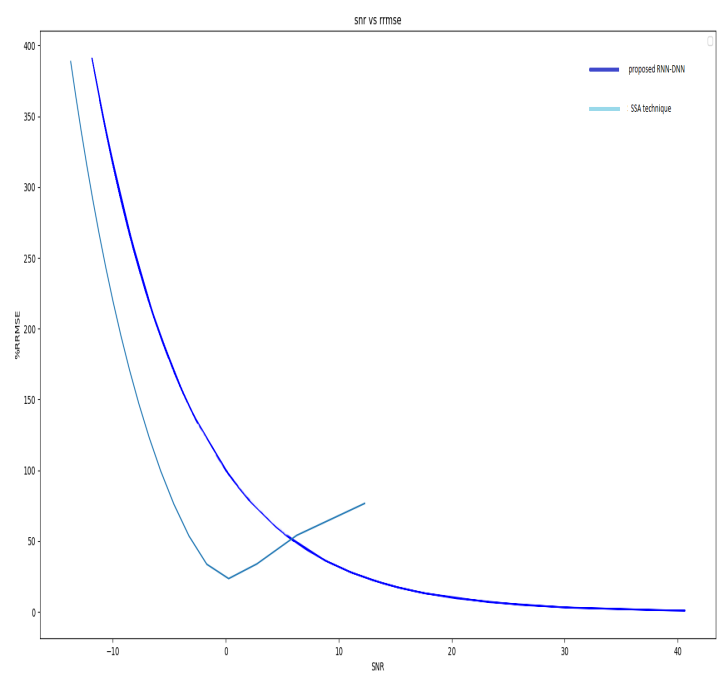

Fig. 11. Comparison of RRMSE curves obtained at different SNR values between $[-10,10]$ using the SSA technique and the proposed RNN-DNN technique.

TABLE II

PERFORMANCE OF MOTION ARTIFACT REMOVAL BETWEEN PROPOSED RNN-DNN AND SSA TECHNIQUES

\begin{tabular}{|l|l|l|}
\hline \multirow{2}{*}{$\begin{array}{l}\text { SNR } \\
(\mathbf{d B})\end{array}$} & \multicolumn{2}{|c|}{ RRMSE(\%) } \\
\cline { 2 - 3 } & $\mathbf{S S A}$ & $\boldsymbol{R} \boldsymbol{N} \boldsymbol{N}-\boldsymbol{D N N}$ \\
\hline 6.2 & 54.06 & 48.42 \\
\hline 6.5 & 57.05 & 47.52 \\
\hline 7.1 & 57.32 & 44.28 \\
\hline 7.5 & 58.64 & 42.32 \\
\hline 8.0 & 60.55 & 40.01 \\
\hline 8.5 & 62.47 & 37.73 \\
\hline 9.0 & 64.28 & 35.48 \\
\hline 9.5 & 66.26 & 33.71 \\
\hline 10.0 & 68.07 & 31.09 \\
\hline 10.5 & 69.95 & 29.98 \\
\hline 11.0 & 71.83 & 28.13 \\
\hline 11.5 & 73.68 & 26.72 \\
\hline 12.2 & 76.63 & 24.81 \\
\hline
\end{tabular}

\section{DiscusSiON}

The as of now proposed strategy gives a completely useful denoising channel for the expulsion of movement artifacts for ECG signals. This strategy can be additionally stretched out for denoising other tainted signals, for example, EEG, PPG, and so forth. To show signs of improvement comprehension of the exhibition of our model we have compared with the past work of others, for instance, with [11] and [14]. In [11], the MSE value for the best case is seen as 0.0073 while the determined MSE for the current technique was 0.00122 with a SNR of $38.7 \mathrm{~dB}$ and in [12], the best case RMSE is recognized to be 0.10 though the RMSE determined for the current strategy is 0.00058 with a SNR of $38.7 \mathrm{~dB}$. As compared by the information given above the current technique seems, by all accounts, to be giving better artifact-free output.

\section{CONCLUSION}

This paper presents a deep learning technique for the expulsion of motion antiquities from ECG signals utilizing the blend of reccurent neural networks and deep neural systems (RNN-DNN). The commotion decrease ability of the proposed technique is tried and approved utilizing ECG signals taken from a standard MIT-BIH Arrhythmia database. The reproduced signals were contrasted and the yield of existing de-noising method singular spectrum analysis (SSA) utilizing SVD. By contrasting the presentation assessment aftereffects of two strategies as appeared in Table II, it could be exhibited that the RNN-DNN based technique acquired higher SNR and less RRMSE than the SSA-based strategy. Later on, the RNN-DNN based technique will be utilized for the continuous ECG signals so as to additionally confirm the adequacy of the motion antiquities expulsion of the proposed strategy.

\section{ACKNOWLEDGEMENT}

The first two authors of this paper acknowledges Department of Electronics and Electrical Engineering at Indian Institute of Technology Guwahati for providing us the summer internship.

\section{REFERENCES}

[1] J. Lee, D. D. McManus, S. Merchant and K. H. Chon, "Automatic Motion and Noise Artifact Detection in Holter ECG Data Using Empirical Mode Decomposition and Statistical Approaches," in IEEE Transactions on Biomedical Engineering, vol. 59, no. 6, pp. 1499-1506, June 2012, doi: 10.1109/TBME.2011.2175729.

[2] A. Karagiannis and P. Constantinou, "Noise-Assisted Data Processing With Empirical Mode Decomposition in Biomedical Signals," in IEEE Transactions on Information Technology in Biomedicine, vol. 15, no. 1, pp. 11-18, Jan. 2011, doi: 10.1109/TITB.2010.2091648.

[3] J. S. Paul, M. R. Reddy and V. J. Kumar, "A transform domain SVD filter for suppression of muscle noise artefacts in exercise ECG's," in IEEE Transactions on Biomedical Engineering, vol. 47, no. 5, pp. 654663, May 2000, doi: 10.1109/10.841337.

[4] C. -. H. Chu and E. J. Delp, "Impulsive noise suppression and background normalization of electrocardiogram signals using morphological operators," in IEEE Transactions on Biomedical Engineering, vol. 36, no. 2, pp. 262-273, Feb. 1989, doi: 10.1109/10.16474.

[5] A. K. Barros, A. Mansour and N. Ohnishi, "Removing artifacts from electrocardio-graphic signals using independent components analysis," Neurocomputing, vol. 22, pp. 173-186, 1998. 
[6] K. Chang, "Arrhythmia ECG noise reduction by ensemble empirical mode decomposition," Sensors, vol. 10, pp. 6063-6080, 2010.

[7] S. Li and J. Lin, "The optimal de-noising algorithm for ECG using stationary wavelet transform," WRI World Cong. on Computer Science and Inform. Eng., vol. 6, pp. $469-473,2009$.

[8] S. Kiranyaz, T. Ince and M. Gabbouj, "Real-Time Patient-Specific ECG Classification by 1-D Convolutional Neural Networks," in IEEE Transactions on Biomedical Engineering, vol. 63, no. 3, pp. 664-675, March 2016, doi: 10.1109/TBME.2015.2468589.

[9] E. D. Obeyli, Combining recurrent neural networks with eigenvector methods for classification of ECG beats, Digital Signal Processing, 19(2), 2009, 320 - 329.

[10] E. D. Obeyli, Recurrent neural networks employing Iyapunov exponents for analysis of ECG signals, Expert Systems with Applications, 37(2), 2010, 1192 - 1199 .

[11] L. Routray, P. Biswal and S. R. Pattanaik, "ECG Artifact Removal of EEG signal using Adaptive Neural Network," 2018 IEEE 13th International Conference on Industrial and Information Systems (ICIIS), Rupnagar, India, 2018, pp. 103-106, doi: 10.1109/ICIINFS.2018.8721423.

[12] N. A. Mohamed, M. Yaseen, W. Hateem, T. Musa and S. Ismail, "Diagnosis of Cardiac Disorders in ECG Signal using Artificial Neural Network Algorithm," 2019 International Conference on Computer, Control, Electrical, and Electronics Engineering (ICCCEEE), Khartoum, Sudan, 2019, pp. 01-07, doi: 10.1109/ICCCEEE46830.2019.9071371.

[13] M. N. Tibdewal and K. B. Thakare, "ANN Based Automatic Identification and Classification of Ocular Artifacts and Non-Artifactual EEG," 2018 Second International Conference on Intelligent Computing and Control Systems (ICICCS), Madurai, India, 2018, pp. 980-985, doi: 10.1109/ICCONS.2018.8663157.

[14] M. Singha Roy, R. Gupta, J. K. Chandra, K. Das Sharma and A. Talukdar, "Improving Photoplethysmographic Measurements Under Motion Artifacts Using Artificial Neural Network for Personal Healthcare," in IEEE Transactions on Instrumentation and Measurement, vol. 67, no. 12, pp. 2820-2829, Dec. 2018, doi: 10.1109/TIM.2018.2829488.

[15] D. Jia, W. Zhao, J. Hu, H. Wang, C. Yan and Z. Li, "Detection of First-Degree Atrioventricular Block on Variable-Length Electrocardiogram via a Multimodal Deep Learning Method," 2019 Computing in Cardiology (CinC), Singapore, Singapore, 2019, pp. Page 1-Page 4, doi: 10.23919/CinC49843.2019.9005846.

[16] D. Kingma and J. Ba, Adam: A method for stochastic optimization, 2014.

[17] Jen-Tzung Chien, Chapter 2 - Model-Based Source Separation, Editor(s): Jen-Tzung Chien, Source Separation and Machine Learning, Academic Press, 2019, Pages 21-52, ISBN 9780128177969, https://doi.org/10.1016/B978-0-12-804566-4.00013-9.

[18] A. K. Maddirala and R. A. Shaik, "Removal of EMG artifacts from single channel EEG signal using singular spectrum analysis," 2015 IEEE International Circuits and Systems Symposium (ICSyS), Langkawi, 2015, pp. 111-115, doi: 10.1109/CircuitsAndSystems.2015.7394075

[19] A. K. Maddirala and R. A. Shaik, "Separation of artifacts from electroencephalogram signal using sequential singular spectrum analysis," 2015 International Conference on Signal Processing and Communication Engineering Systems, Guntur, 2015, pp. 384-388, doi: 10.1109/SPACES.2015.7058290.

[20] A. K. Maddirala and R. A. Shaik, "Separation of Sources From SingleChannel EEG Signals Using Independent Component Analysis," in IEEE Transactions on Instrumentation and Measurement, vol. 67, no. 2, pp. 382-393, Feb. 2018, doi: 10.1109/TIM.2017.2775358.

[21] A. K. Maddirala and R. A. Shaik, "Removal of EOG Artifacts From Single Channel EEG Signals Using Combined Singular Spectrum Analysis and Adaptive Noise Canceler," in IEEE Sensors Journal, vol. 16, no. 23, pp. 8279-8287, Dec.1, 2016, doi: 10.1109/JSEN.2016.2560219.

[22] https://www.physionet.org/content/mitdb/1.0.0/

[23] https://towardsdatascience.com/a-laymans-guide-to-deep-neuralnetworks-ddcea $24847 \mathrm{fb}$ 